\title{
La expresión oral en primaria: A propósito de una experiencia práctica en el aula*
}

\author{
Luis Ángel Prieto Carnicero**, Isabel Cantón Mayo***
}

\begin{abstract}
RESUMEN
El objetivo general de este trabajo es evaluar el nivel de expresión oral en la competencia lingüística adquirido por el alumnado de Educación Primaria. Asimismo, se pretende analizar la influencia que pudieran tener variables como el género y el tipo de centro. La muestra está compuesta por un total de 60 individuos, que presentan una edad comprendida entre los 9 y los 10 años, a quienes se les aplicó una prueba ad hoc de expresión oral, a través de una ilustración. Entre los resultados se destaca que la expresión oral del alumnado se encuentra en un nivel medio.
\end{abstract}

Palabras clave: Expresión oral, educación primaria, competencia lingüística.

\section{The oral expression in Primary Education: A practical experience in the classroom}

\begin{abstract}
The overall objective of this study is to evaluate the level of oral expression in the language skills acquired by Primary Education students. It also aims to analyze the influence of variables such as gender and type of school. The total sample consists of 60 individuals between 9 and 10 years old, who passed an ad hoc test of oral expression through an illustration. Among other results, their oral expression is at a medium level.
\end{abstract}

Keywords: Oral expression, primary education, language proficiency.

* Este artículo presenta los resultados parciales contenidos en una investigación mayor: Prieto, L. (2012). Evaluación de la competencia lingǘstica en alumnos de $4^{\circ}$ de Primaria (tesis de doctorado). España: Universidad de León.

* Luis Ángel Prieto Carnicero es Doctor en Psicología y Ciencias de la Educación y Licenciado en Lingüística de la Universidad de León, España-. E-mail: theroyalbritishempire@gmail.com

* Isabel Cantón Mayo es Catedrática de Didáctica y Organización Escolar de la Universidad de León, España. E-mail: icanm@unileon.es 


\section{Introducción}

"Hablar es tener algo que decir y saber decirlo; encontrar las palabras, construir bien las frases, darles su propia expresión. Entonces es cuando la comunicación es fácil, considerar siempre como un todo lo que se dice, cómo se dice y a quién se dice" (Aymerich y Aymerich, I97I, p. 6I). La escuela recoge la importancia de esta destreza, pues la expresión oral contribuye, en el currículo del área de Lengua y Literatura, al desarrollo del pensamiento y la competencia lingüística (Serra, Serrat, Solé, Bel y Aparici, 2013; Vygotsky, 2012; Pinker, 2003). Así, el Real Decreto I5I3/2006, de 7 de diciembre, por el que se establecen las enseñanzas mínimas de la Educación Primaria (Ministerio de Educación, 2006), apunta que la enseñanza de la lengua y literatura, en esta etapa, tendrá entre sus objetivos el desarrollo de las capacidades de comprensión y expresión oral y escrita; el uso adecuado de la lengua en diferentes contextos; así como el empleo de la lengua como medio de relación social y cultural y control de la propia conducta.

El aprendizaje de la oralidad, como contenido educativo en las aulas, ha evolucionado. Desde un primer período, comprendido entre el Plan Pidal de I845 y la Ley de I970, donde la dimensión oral no tenía cabida, hasta la llegada de los diseños curriculares elaborados a partir de la Ley Orgánica General del Sistema Educativo (I990), la cual pasa a recoger una nueva perspectiva y reestructuración de los contenidos en los que se comienza a tener en cuenta el enfoque comunicativo y la oralidad (Lomas, 2015; Quiles, 2006). Sin embargo, no parece muy seguro que esos cambios hayan traspasado el simple nivel abstracto de la definición de objetivos y de las buenas intenciones para alcanzar los cambios efectivos que la práctica requiere en los distintos niveles educativos, puesto que "el tratamiento de la lengua oral formal en las aulas sigue siendo minoritario y escaso (...)” (Vilá y Castellá, 20I4, p. II).

Se observa que la escuela no se olvida de la importancia que hechos como estos tienen para el desarrollo integral de la persona, sobre todo, en su nivel de competencia lingüística. Sin embargo, ya desde las aulas de Primaria de nuestro sistema educativo, voluntaria o involuntariamente, los alumnos desempeñan el papel 
de espectadores, más que el de actores, de su propia competencia lingüística oral.

A pesar del reconocimiento que se presta a la oralidad en el currículo oficial, serán los contenidos conceptuales los que predominen en la labor docente, relegando la expresión oral a actividades no estructuradas y sin planificación previa, en el currículo oculto. La carencia de experiencias y metodologías (Vila y Vila, 1994); la presión por completar un currículo fijado de antemano y, la falta de unas orientaciones metodológicas concretas por parte de la administración educativa obligan, generalmente, a prescindir de la expresión oral en las aulas. Y, mientras tanto, el alumno no aprenderá a escuchar, a hablar ni a conversar, confiando en que la adquisición de la competencia comunicativa oral la aprenda del modelo que constituye el docente (Alférez, 2015; Segovia, Gallego y Rodríguez, 2013; Cruzata-Guzmán y Salazar-Salazar, 2012). Por tal motivo, en la práctica escolar se han afianzado las formas de práctica escrita, mientras que las orales se limitan a la explicación magistral, a la repetición y a la resolución de dudas. Zuccherini (1992) señala algunas de las razones de la resistencia escolar al lenguaje hablado:

I. Desde el punto de vista educativo, la lengua oral presenta muchas dificultades, sobre todo, cuando se la compara con las posibilidades que ofrece el texto escrito (permanencia, corregibilidad, reflexión, dictabilidad, uniformidad, etc.). El texto oral, por el contrario, limita la reflexión profunda y puntual, meticulosa, que permite el escrito: resulta difícil recordar las palabras y frases en la forma exacta en que se han producido, y es aún más difícil intentar cambiar, corregir y mejorar el texto oral.

2. Así mismo, los niños, principalmente los de menor edad, no siguen durante mucho tiempo un trabajo de reflexión sobre la lengua oral que, por cansancio y distracción, puede volverse pesado y contraproducente.

Además de las razones anteriormente citadas, podrían ser sumadas muchas otras, diversas y de distinta naturaleza (Gutiérrez y Rosas, 2008). Por nuestra parte, se incluyen las siguientes: 
I. Los esfuerzos pedagógicos se centran en la lengua escrita, ante la creencia de que quien domina el vocabulario, la sintaxis, la expresión escrita, será capaz de transferirlo al habla. Según esto, ¿se debiese pensar que Cervantes, por haber escrito su obra más conocida, El Quijote, dominaba la expresión oral con igual destreza, al punto de ser un gran orador?

2. Los hablantes, por el hecho de saber hablar su lengua materna, pueden prescindir de refuerzos externos para el aprendizaje. En un claro paralelismo, podría decirse que cualquiera es un atleta de élite solo por el hecho mismo de saber caminar.

Como consecuencia del anteriormente mencionado currículo oculto, la expresión oral no se aplica en el área de Lengua con tanta frecuencia como desearían los alumnos, impidiendo así su estimulación (Gómez, 2013). Generalmente, los alumnos tienen interés en las actividades de expresión oral. Tal interés, además, viene reforzado por la necesidad que los niños tienen de expresar sus experiencias a los demás y de relacionarse con el otro. Las aulas se componen de un nuevo modelo de alumno que, acostumbrado a una mayor frecuencia de relaciones, tanto en el ámbito escolar como en el extraescolar, la extraversión se generaliza y, fruto de ello, la socialización a través de la comunicación oral.

Las razones de esa escasa frecuencia podrían encontrarse en un desplazamiento de las actividades de expresión oral a otras áreas curriculares, que favorecen el intercambio de ideas (Conocimiento del Medio, por ejemplo). Tal afirmación revela una contradicción en los términos: si la clase de Lengua es el área encargada principal de impartir la expresión oral, ¿̨por qué no se realizan en ella actividades en las que los alumnos tengan conciencia de que están aplicando los conocimientos teóricos aprendidos?

En el discurso de la educación en competencias (Moya y Luengo, 20I I; Doncel y Leena, 20I I; Gimeno et al., 2008; Marco, 2008), la importancia que tiene la expresión oral en la competencia lingüística y la apertura que se ha ido produciendo entre los individuos de una misma sociedad y entre sociedades diferentes, hace que sea necesario un mayor aprendizaje y dominio de tal destreza. Por lo tanto, 
conscientes de tal importancia, este estudio pretende evaluar el nivel alcanzado por los alumnos en expresión oral. Aunque el objetivo de la investigación podría ser aplicado en cualquier grado de Primaria, se ha optado por el $4^{\circ}$, por ser esta una etapa intermedia en ella y por permitir poner sus resultados en relación con otros estudios, tales como PISA, por ejemplo, que si bien este no tiene el compromiso de analizar la expresión oral, no deja de existir un vínculo directo entre la competencia lectora y la abstracción de ideas y argumentos que requiere la comunicación lingüística.

\section{Objetivos}

\subsection{Objetivo general}

Evaluar el nivel de competencia lingüística, en su vertiente oral descriptiva, del alumnado de $4^{\circ}$ de Primaria.

\subsection{Objetivos específicos}

I. Identificar las diferencias significativas en la consecución de los objetivos de expresión oral entre alumnos y alumnas.

2. Estimar la influencia que el tipo de centro pueda tener en la adquisición de la expresión oral del alumno.

\section{Método}

\subsection{Participantes}

La población a la que se dirige esta investigación está conformada por el alumnado de $4^{\circ}$ curso de Educación Primaria de todos los centros públicos o privados que se encuentran localizados en el área geográfica de León y su provincia.

La muestra está compuesta por un total de 60 individuos, que presentan una edad comprendida entre los 9 y los 10 años. Se obtiene de la selección de 4 alumnos de entre las aulas de $4^{\circ}$ de Educación Primaria de cada uno de los centros visitados. 
En cuanto al género de los alumnos participantes, se compone de $3 \mathrm{I}$ hombres y de 29 mujeres, lo que se corresponde con un 5I,6\% y un $48,3 \%$, respectivamente, de la muestra resultante. Esta variable fue controlada en la selección de los individuos, pues se buscaba que la proporción de ambos géneros fuera equilibrada, o igual, a ser posible.

El estrato está formado por los centros públicos y privados de Primaria y, dentro de ellos, los urbanos y los rurales. No se ha podido determinar el estrato centro privado rural, dado que en la provincia de León no se encuentra ninguna población de menos de 10.000 habitantes $\left(<\mathrm{I} 0.000\right.$ habitantes $=$ núcleo rural, según $\left.\mathrm{INE}^{\mathrm{I}}\right)$ que cuente con un centro escolar de tales características. Así mismo, para la selección de la muestra de los centros públicos rurales se ha tenido en cuenta que existiera una representación proporcional de los cuatro puntos geográficos (norte, sur, este y oeste) dentro de los límites de la provincia de estudio. La selección de los centros -y, en consecuencia, del alumnado- se ha realizado de manera aleatoria.

El número de centros necesarios para intervenir en la investigación fue establecido en I5, y atendiendo a su estratificación, en la que cada uno de esos estratos recogiera una parte proporcional; en este caso, 5 centros por cada estrato.

\subsection{Instrumentos}

Para la recogida de datos a los alumnos se les aplicó una prueba ad boc de expresión oral, a través de una ilustración. Con el material de evaluación se acompaña una plantilla, de utilidad para el administrador del instrumento de medida. Presenta una escala de medida ordinal, realizada por la persona encargada de administrar la prueba. Es una escala Likert en forma descriptiva, de 4 grados dentro de un continuo. Las posibilidades de respuesta se presentan en forma de alternativas con una disposición descendente en la gradación, siendo muy bueno/siempre para el mayor grado y malo/nunca para el inferior. Los ítems se distribuyen en dos bloques. Esta división sirve para realizar por separado el análisis de los resultados obtenidos en

INE corresponde a la sigla del Instituto Nacional de Estadísticas de España. 
cada una de las categorías (Análisis de contenido y Análisis formal). Para determinar el nivel de expresión oral en el que se encuentra cada alumno se debe tener en cuenta la puntuación alcanzada en la suma total de todos los ítems.

Cada una de las respuestas dadas en los ítems se corresponde con una puntuación. Si se considera la respuesta (I) la puntuación es de I punto, con la respuesta (2) se otorga 0,5 puntos, con la respuesta (3) la puntuación es de 0,25 puntos y con la última respuesta (4) no se concede punto alguno ( 0 puntos). Sumados los puntos resultantes en cada ítem, se dispone de la puntuación final del alumno evaluado, el cual será incluido en una escala, que recoge distintos niveles de expresión oral, dependiendo de la puntuación final obtenida.

\subsection{Procedimiento}

Se recogieron un total de 60 pruebas realizadas por los alumnos de los distintos centros. A los 4 alumnos participantes de cada una de las I5 aulas se les pide que realicen la descripción detallada de una ilustración infantil, a color, que representa una escena de la vida cotidiana. Antes de comenzar recibieron las instrucciones, individualmente, de idéntica manera. Se permite la descripción continuada, sin interrupciones. Sus descripciones se recogen en soporte técnico para su posterior transcripción y registro informático. Los datos resultantes han sido introducidos en una plantilla informática ofrecida por el Paquete Estadístico SPSS en su versión I 5.0 para Windows. En esa plantilla se ha definido cada una de las variables de contenido y variables de forma y se determinaron sus valores correspondientes. Las variables de forma que requieren el análisis acústico de los sonidos del habla, fueron analizadas mediante el programa informático Speech Analyzer 3.I. Se crearon diccionarios que permitieran el recuento de léxico, densidad semántica, etc.

Se llevó a cabo un análisis factorial de los ítems, a través de la matriz de correlaciones, su comunalidad, la varianza total explicada, la matriz de componentes, la matriz de componentes rotados y la matriz de transformación de las componentes. Como prueba complementaria, dentro de la matriz de correlaciones, el índice KMO y la prueba de 
esfericidad de Bartlett. El método de extracción empleado ha sido mediante el Análisis de Componentes Principales y para el método de rotación la Normalización Varimax. A continuación, se utilizó el coeficiente alpha de Cronbach y la técnica de las dos mitades, para estudiar la consistencia interna.

\section{Resultados}

\subsection{Análisis de contenido}

Un 45\% de alumnos comienzan su exposición oral sin definir el marco en el que se desarrolla la acción que describen (Tabla I).

Tabla I.Análisis de contenido. Resultados

\begin{tabular}{|c|c|c|c|c|c|}
\hline & & Siempre & $\begin{array}{l}\text { Bastantes } \\
\text { veces }\end{array}$ & A veces & Nunca \\
\hline \multirow{12}{*}{ 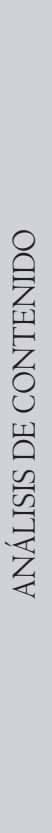 } & $\begin{array}{l}\text { Comienza enmarcando lo que va } \\
\text { a describir }\end{array}$ & $33,3 \%$ & $10,0 \%$ & $\mathrm{II}, 7 \%$ & $45,0 \%$ \\
\hline & $\begin{array}{l}\text { Hay una buena selección de } \\
\text { detalles }\end{array}$ & $10,0 \%$ & $30,0 \%$ & $4 \mathrm{I}, 7 \%$ & $18,3 \%$ \\
\hline & Evita los elementos superfluos & $58,3 \%$ & $25,0 \%$ & $13,3 \%$ & $0,0 \%$ \\
\hline & $\begin{array}{l}\text { Descripción sin vueltas atrás, líos, } \\
\text { etc. }\end{array}$ & $76,7 \%$ & $10,0 \%$ & $10,0 \%$ & $0,0 \%$ \\
\hline & $\begin{array}{l}\text { Utiliza adjetivación (color, forma, } \\
\text { tamaño...) }\end{array}$ & $\mathrm{I}, 7 \%$ & I,7\% & $8,3 \%$ & $88,3 \%$ \\
\hline & Los adjetivos son precisos & $10,0 \%$ & $0,0 \%$ & $1,7 \%$ & $88,3 \%$ \\
\hline & $\begin{array}{l}\text { Utiliza localizadores espaciales } \\
\text { (arriba, abajo, derecha, etc.) }\end{array}$ & $\mathrm{I}, 7 \%$ & $6,7 \%$ & $26,7 \%$ & $65,0 \%$ \\
\hline & $\begin{array}{l}\text { Evita repetir, insistentemente, las } \\
\text { mismas palabras }\end{array}$ & $28,3 \%$ & $50,0 \%$ & $21,7 \%$ & $0,0 \%$ \\
\hline & Describe con objetividad & $61,7 \%$ & $16,7 \%$ & $21,7 \%$ & $0,0 \%$ \\
\hline & Existe unidad en la descripción & $18,3 \%$ & $33,3 \%$ & $43,3 \%$ & $5,0 \%$ \\
\hline & Utiliza frases subordinadas & $3,3 \%$ & $23,3 \%$ & $65,0 \%$ & $8,3 \%$ \\
\hline & Es una descripción completa & $10,0 \%$ & $3 \mathrm{I}, 7 \%$ & $45,0 \%$ & $\mathrm{I} 3,3 \%$ \\
\hline
\end{tabular}

Otro 4I,7\% concede poca importancia a los detalles, eligiendo solo los que les parecen más destacados, sin completarlos todos. E1 $58,3 \%$ evita los elementos superfluos en su exposición; mientras que el 
$76,7 \%$ realiza una descripción sin vueltas atrás, líos o cualquier otro hecho que dificulte su exposición. En cuanto al uso de adjetivación, resulta curioso comprobar que el $88,3 \%$ de los alumnos no utilizaran ningún adjetivo, si se tiene en cuenta que la ilustración presenta gran variedad de colores en los que resulta imposible que el alumno no repare, y si consideramos que el uso del adjetivo es muy trabajado en el aula de Lengua. E1 65\% nunca utiliza localizadores espaciales y el 26,7\% lo hace a veces, incluyendo alguno. Evitan repetir las mismas palabras el 50\%, y un 28,3\% lo cumple siempre. Podría afirmarse que cualquier repetición se debe más a la tensión que les pudiera provocar la situación, que a la falta de un repertorio lingüístico más o menos desarrollado.

Por otra parte, se presenta un 61,7\% de alumnos que permanecen ajenos a la acción que se desarrolla, y describen con objetividad. Existe un 5I,6\% (siempre + bastantes veces) que consigue darle una unidad a su descripción. Mientras tanto, el uso de oraciones subordinadas es poco frecuente $(65 \%$, a veces). El tipo de oraciones subordinadas más frecuente son las adjetivas o de relativo $y$, dentro de ellas, las explicativas. De igual manera, emplean frecuentemente las subordinadas adverbiales, tanto las de lugar, modo o final. Hacen un mayor uso de la coordinación, sobre todo, de las oraciones copulativas. Finalmente, las descripciones resultan completas en un 41,7\% (siempre + bastantes veces). En ocasiones, estas descripciones se convierten en simples enumeraciones de objetos o de personas, sin expresar qué papel desempeñan aquéllos en el contexto o qué posición ocupan dentro del mismo.

\subsubsection{Análisis de contenido por género}

El $54,8 \%$ de los hombres nunca comienzan enmarcando lo que van a describir (Tabla 2). Por otro lado, cuando hay que iniciar enmarcando lo que se va a explicar, son las mujeres en un 51,7\% (siempre + bastantes veces) las que lo hacen, en proporción mayor y significativa que el $35,5 \%$ de los hombres. Son los hombres, con un $45,2 \%$ (siempre + bastantes veces), quienes realizan una buena selección de detalles, y las mujeres $(44,8 \%)$ cuando se trata de una selección no tan completa (a veces). 
Si de evitar los elementos superfluos se trata se presenta una simetría entre ambos grupos (bombres: 58,1\% / mujeres: 58,6\%), aunque sean las mujeres las que estén mínimamente por encima. Las diferencias entre hombres y mujeres para hacer una exposición clara, sin líos, vueltas atrás o embrollos se presenta en un porcentaje mayor de mujeres $(82,8 \%)$, marcando diferencias con respecto al 7I\% de los hombres.

Ya se expuso más arriba que el uso de adjetivos y de localizadores espaciales era algo poco frecuente en el conjunto de las exposiciones orales, en general. Por géneros sucede lo mismo, siendo limitado su empleo tanto en hombres como mujeres. No obstante, serán los hombres con el $93,5 \%$ y el $71 \%$, quienes nunca hagan uso de la adjetivación y localizadores, respectivamente.

Ambos géneros mantienen una simetría al evitar repetir constantemente las mismas palabras. Sin embargo, serán las mujeres las que formulen una descripción más objetiva $(75,9 \%)$ y consigan no interferir con su punto de vista en la descripción que realizan. En cuanto a la existencia de unidad en la descripción, el uso de frases subordinadas y formular una descripción completa, no existen diferencias significativas entre ambos grupos.

Tabla 2. Análisis de contenido. Resultados por género

\begin{tabular}{|c|c|c|c|c|c|c|}
\hline & & & Siempre & $\begin{array}{c}\text { Bastantes } \\
\text { veces }\end{array}$ & A veces & Nunca \\
\hline \multirow{12}{*}{ 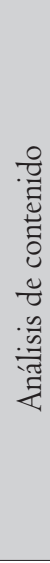 } & \multirow{2}{*}{$\begin{array}{l}\text { Comienza enmarcando lo } \\
\text { que va a describir }\end{array}$} & Hombre & $32,3 \%$ & $3,2 \%$ & $9,7 \%$ & $54,8 \%$ \\
\hline & & Mujer & $34,5 \%$ & $17,2 \%$ & $\mathrm{I} 3,8 \%$ & $34,5 \%$ \\
\hline & \multirow{2}{*}{$\begin{array}{l}\text { Hay una buena selección de } \\
\text { detalles }\end{array}$} & Hombre & $12,9 \%$ & $32,3 \%$ & $38,7 \%$ & $16,1 \%$ \\
\hline & & Mujer & $6,9 \%$ & $27,6 \%$ & $44,8 \%$ & $20,7 \%$ \\
\hline & \multirow{2}{*}{$\begin{array}{l}\text { Evita los elementos } \\
\text { superfluos }\end{array}$} & Hombre & $58,1 \%$ & $19,4 \%$ & $22,6 \%$ & $0,0 \%$ \\
\hline & & Mujer & $58,6 \%$ & $31,0 \%$ & $3,4 \%$ & $6,9 \%$ \\
\hline & \multirow{2}{*}{$\begin{array}{l}\text { Descripción sin vueltas } \\
\text { atrás, líos, etc. }\end{array}$} & Hombre & $71,0 \%$ & $16,1 \%$ & $9,7 \%$ & $3,2 \%$ \\
\hline & & Mujer & $82,8 \%$ & $3,4 \%$ & $10,3 \%$ & $3,4 \%$ \\
\hline & \multirow{2}{*}{$\begin{array}{l}\text { Utiliza adjetivación (color, } \\
\text { forma, tamaño...) }\end{array}$} & Hombre & $0,0 \%$ & $0,0 \%$ & $6,5 \%$ & $93,5 \%$ \\
\hline & & Mujer & $3,4 \%$ & $3,4 \%$ & $10,3 \%$ & $82,8 \%$ \\
\hline & \multirow{2}{*}{ Los adjetivos son precisos } & Hombre & $6,5 \%$ & $0,0 \%$ & $0,0 \%$ & $93,5 \%$ \\
\hline & & Mujer & $\mathrm{I} 3,8 \%$ & $0,0 \%$ & $3,4 \%$ & $82,8 \%$ \\
\hline
\end{tabular}


Tabla 2. Análisis de contenido. Resultados por género (continuación)

\begin{tabular}{|c|c|c|c|c|c|c|}
\hline & & & Siempre & $\begin{array}{c}\text { Bastantes } \\
\text { veces }\end{array}$ & A veces & Nunca \\
\hline \multirow{12}{*}{ 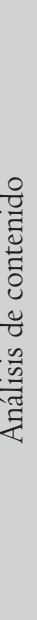 } & \multirow{2}{*}{$\begin{array}{l}\text { Utiliza localizadores } \\
\text { espaciales (arriba, abajo, } \\
\text { derecha, etc.) }\end{array}$} & Hombre & $3,2 \%$ & $6,5 \%$ & $19,4 \%$ & $71,0 \%$ \\
\hline & & Mujer & $0,0 \%$ & $6,9 \%$ & $34,5 \%$ & $58,6 \%$ \\
\hline & \multirow{2}{*}{$\begin{array}{l}\text { Evita repetir, } \\
\text { insistentemente, las mismas } \\
\text { palabras }\end{array}$} & Hombre & $22,6 \%$ & $54,8 \%$ & $22,6 \%$ & $0,0 \%$ \\
\hline & & Mujer & $34,5 \%$ & $44,8 \%$ & $20,7 \%$ & $0,0 \%$ \\
\hline & \multirow{2}{*}{ Describe con objetividad } & Hombre & $48,4 \%$ & $19,4 \%$ & $32,3 \%$ & $0,0 \%$ \\
\hline & & Mujer & $75,9 \%$ & $13,8 \%$ & $10,3 \%$ & $0,0 \%$ \\
\hline & \multirow{2}{*}{$\begin{array}{l}\text { Existe unidad en la } \\
\text { descripción }\end{array}$} & Hombre & $19,4 \%$ & $32,3 \%$ & $4 \mathrm{I}, 9 \%$ & $6,5 \%$ \\
\hline & & Mujer & $17,2 \%$ & $34,5 \%$ & $44,8 \%$ & $3,4 \%$ \\
\hline & \multirow{2}{*}{ Utiliza frases subordinadas } & Hombre & $0,0 \%$ & $25,8 \%$ & $67,7 \%$ & $6,5 \%$ \\
\hline & & Mujer & $6,9 \%$ & $20,7 \%$ & $62,1 \%$ & $10,3 \%$ \\
\hline & \multirow{2}{*}{ Es una descripción completa } & Hombre & $\mathrm{I} 2,9 \%$ & $29,0 \%$ & $45,2 \%$ & $12,9 \%$ \\
\hline & & Mujer & $6,9 \%$ & $34,5 \%$ & $44,8 \%$ & $13,8 \%$ \\
\hline
\end{tabular}

\subsection{Análisis formal}

Una clara articulación se encuentra en el 70\% de los casos (siempre), mientras que otro $25 \%$ presenta un nivel bastante adecuado (bastantes veces) (Tabla 3). El ritmo en las palabras emitidas es muy adecuado en un $51,7 \%$, viéndose alterado en un $28,3 \%$ (bastantes veces) por algún recurso que origina una interrupción (duda, alargamiento de sílabas finales, etc.). El volumen, en el 58,3\% (siempre) y para un 36,7\% (bastantes veces) es el esperado para una voz infantil, que tiende a ser más aguda que una voz ya formada.

La entonación, a pesar de producirse en situación diferente a las rutinas escolares y por persona distinta al docente habitual, se cumple en un $45 \%$ y en otro $30 \%$ ocurre bastantes veces. El nivel del lenguaje es ajustado a una situación ordinaria protagonizada por el alumno, pero sin perder un carácter académico, propio del entorno en el que se desarrolla la acción (86,6\%, siempre + bastantes veces). La expresión es viva y natural en el $45 \%$, puntuación aceptable, si se tiene en cuenta que la situación y el examinador eran desconocidos para los alumnos. 
Tabla 3. Análisis formal. Resultados

\begin{tabular}{|c|c|c|c|c|c|}
\hline & & Siempre & $\begin{array}{c}\text { Bastantes } \\
\text { veces }\end{array}$ & A veces & Nunca \\
\hline \multirow{12}{*}{ 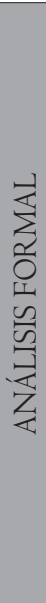 } & Articulación clara & $70,0 \%$ & $25,0 \%$ & $3,3 \%$ & $\mathrm{I}, 7 \%$ \\
\hline & Ritmo adecuado & $51,7 \%$ & $28,3 \%$ & $18,3 \%$ & $1,7 \%$ \\
\hline & Volumen adecuado & $58,3 \%$ & $36,7 \%$ & $3,3 \%$ & $\mathrm{I}, 7 \%$ \\
\hline & Entonación natural y amena & $45,0 \%$ & $30,0 \%$ & $18,3 \%$ & $6,7 \%$ \\
\hline & Nivel del lenguaje adecuado & $43,3 \%$ & $43,3 \%$ & $13,3 \%$ & $0,0 \%$ \\
\hline & Expresión viva y natural & $45,0 \%$ & $23,3 \%$ & $23,3 \%$ & $8,3 \%$ \\
\hline & Léxico abundante y adecuado & $\mathrm{I} 8,3 \%$ & $60,0 \%$ & $18,3 \%$ & $3,3 \%$ \\
\hline & $\begin{array}{l}\text { Morfosintaxis. Estructuras bien } \\
\text { construidas }\end{array}$ & $56,7 \%$ & $33,3 \%$ & $10,0 \%$ & $0,0 \%$ \\
\hline & Fonética: Producción correcta de sonidos & $90,0 \%$ & $6,7 \%$ & $3,3 \%$ & $0,0 \%$ \\
\hline & Habla sin tartamudear & $88,3 \%$ & $8,3 \%$ & $\mathrm{I}, 7 \%$ & $1,7 \%$ \\
\hline & Acompaña su expresión oral con gestos & $\mathrm{I}, 7 \%$ & $5,0 \%$ & $\mathrm{II}, 7 \%$ & $81,7 \%$ \\
\hline & Se muestra relajado cuando habla & $25,0 \%$ & $16,7 \%$ & $41,7 \%$ & $16,7 \%$ \\
\hline
\end{tabular}

En cuanto al léxico utilizado suele ser adecuado a los objetos que quieren definir, pero la abundancia y diversidad léxica no aparecen, llegándose a hacer la descripción de algún detalle con las mínimas palabras. Las estructuras lingüísticas, siendo sencillas, están bien construidas (56,7\%), pudiéndose encontrar algunos errores morfológicos muy comunes (contracción de palabras, por ejemplo). La fonética de los alumnos es correcta (90\%), incluso, en los fonemas con cierta dificultad /rr/. No aparece tartamudeo (88,3\%), sin llegar a ser una patología lingüística en el restante II,3\%, sino vacilaciones y correcciones, que producen un efecto entrecortado, similar al tartamudeo. La expresión gestual es muy limitada, tendiendo a apoyar las manos sobre la mesa o colocándolas detrás de su espalda. Se acompaña algún tímido gesto en el aire o señalando la ilustración. En general, existe un nivel de relajación medio mientras hablan (4I,7\%). Únicamente, un I6,7\% muestra claros síntomas de encontrarse incómodo y tenso (velocidad discursiva, movimientos incontrolados de sus manos).

\subsubsection{Análisis formal por género}

La articulación de las mujeres es más clara $(75,9 \%)$ de una manera constante (siempre) (Tabla 4). Son los hombres los que presentan un buen ritmo $(54,8 \%)$ y volumen $(64,5 \%)$ por encima de las mujeres (siempre). 
Aunque serán las mujeres las que alcancen el I00\% de los casos, si se atiende al nivel superior completo (siempre + bastantes veces). Los casos de un volumen inadecuado se corresponden con casos de hombres.

Los hombres consiguen una entonación más natural y amena en el 77,4\% de los sujetos (siempre + bastantes veces), no muy superior al 72,4\% de las mujeres. Tampoco se presentan significativas distancias en el nivel superior (siempre + bastantes veces) al observar el nivel del lenguaje (bombres: 83,7\%/mujeres: 89,7\%); la viveza y naturalidad de la expresión (bombres: 7I\%/mujeres: 65,5\%); y la abundancia y adecuación del léxico (bombres: 80,6\%/mujeres: 75,9\%).

Tabla 4. Análisis formal. Resultados por género

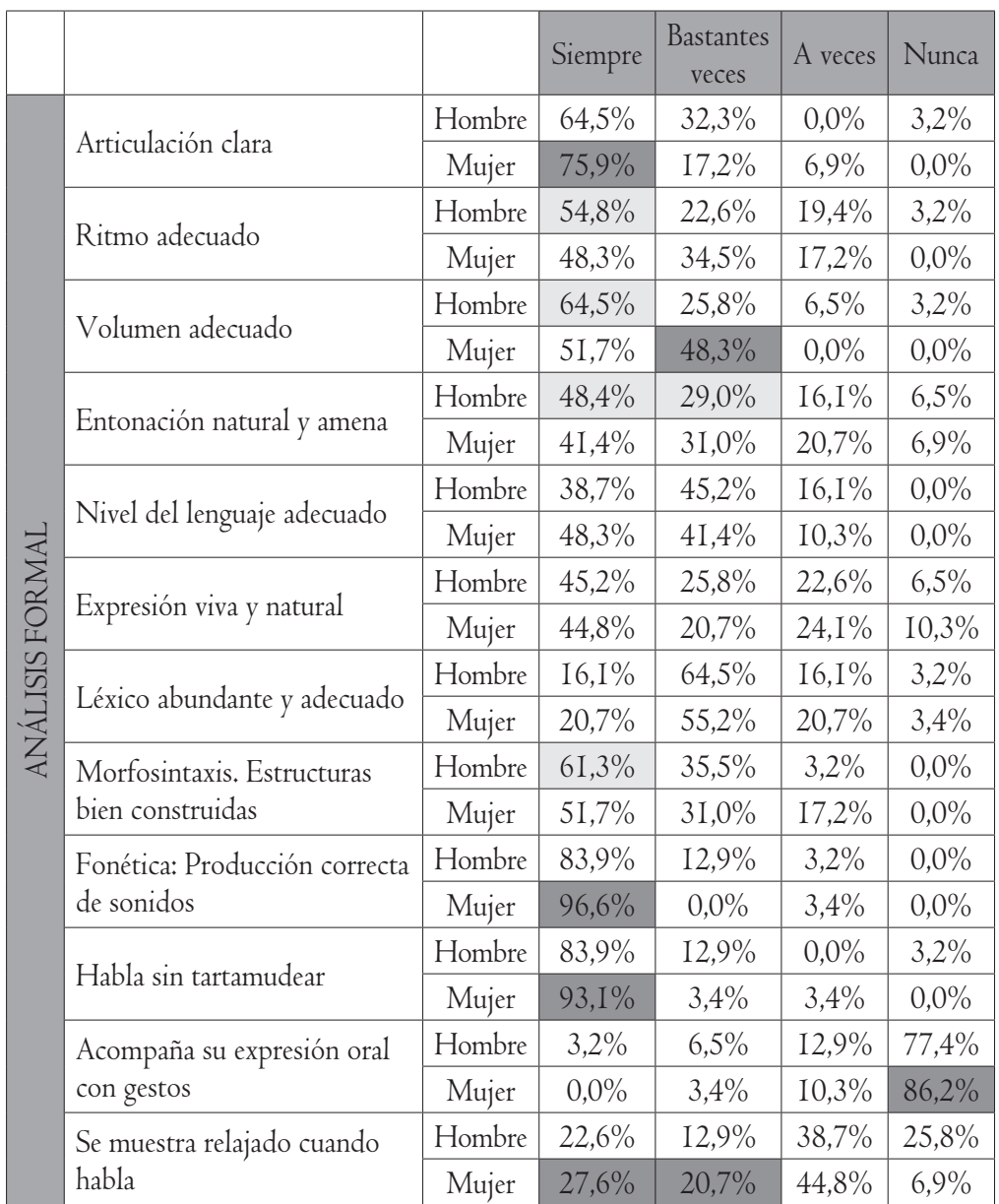


En morfosintaxis fueron los hombres (96,8\%) los que elaboraron unas mejores construcciones (siempre + bastantes veces). Mientras que en fonética y un discurso sin tartamudear no se aprecian diferencias entre géneros y, sí aparecen aquéllas en la expresión gestual, pues, dentro de los resultados generales, son menos expresivas las mujeres (nunca: $86,2 \%$ ) frente a los hombres (nunca: 77,4\%). Este control gestual de las mujeres en sus movimientos puede ser la consecuencia de tener, también, un mayor control corporal traducido en una mayor relajación mientras hablan. Así, casi la mitad de las mujeres (siempre + bastantes veces $=48,3 \%$ ) se muestran más relajadas mientras hablan, que los hombres (35,5\%) en el mismo nivel superior.

\subsection{Resultados generales: Niveles de expresión oral}

Después de ser sumadas las puntuaciones resultantes en el Análisis de Contenido y en el Análisis Formal, se obtiene la distribución de la muestra en los diferentes niveles de expresión oral. De este modo, el nivel que mayor frecuencia alcanza se corresponde con el nivel dentro de la normalidad (Figura I). Aquí, se encuentra el 45\% de los alumnos de la muestra ( $\mathrm{n}=27$ sujetos). Este nivel representa la línea media de las puntuaciones. En menor proporción, aunque a destacar, se encuentra el $2 \mathrm{I}, 7 \%$ de los alumnos ( $\mathrm{n}=\mathrm{I} 3$ sujetos) dentro del nivel moderadamente alto. Pocos se posicionan en los niveles de excelencia (alto: $\mathrm{n}=2$ sujetos; $3,3 \%$ ). Por el contrario, algo más del 30\% ( $\mathrm{n}=18$ sujetos) permanece entre los niveles inferiores.

Figura I. Expresión oral. Niveles de puntuación
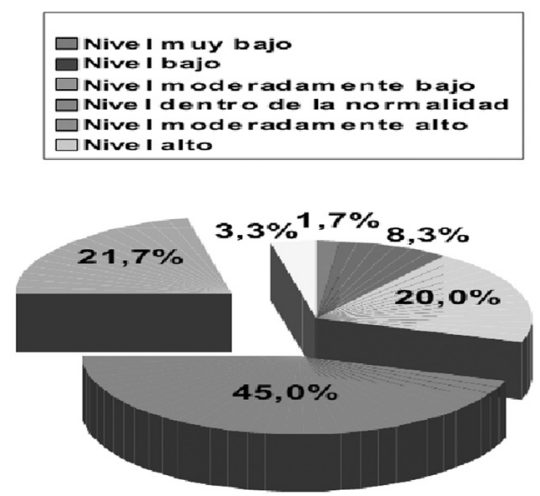
Por géneros, puntúan los hombres en el nivel dentro de la normalidad con un $48,4 \%$, por encima del 4I,4\% obtenido por las mujeres (Figura 2). Sucederá, sin embargo, lo contrario entre el nivel moderadamente alto. Las mujeres aglutinan una proporción mayor (24,1\%) que el 19,4\% de los hombres. También, será el 6,9\% de las mujeres las que se encuentren entre el nivel alto, en esta ocasión sin representación masculina en dicho nivel. En los niveles más extremos (alto y sin nivel) no se ha producido ningún caso. Tanto hombres como mujeres alcanzan puntuaciones más altas en el Análisis Formal que en el Análisis de Contenido.

Figura 2. Expresión oral. Puntuaciones finales por género

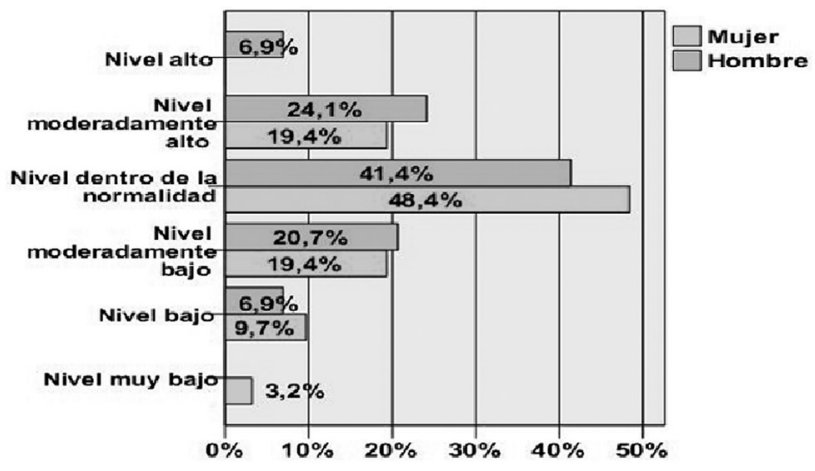

Según el tipo de centro, en el nivel dentro de la normalidad se presentan las mayores frecuencias para los tres tipos de centros. Serán los privados urbanos los que destaquen con un I8\% sobre los públicos rurales y los públicos urbanos, cada uno con un I3\% del total de la muestra (Figura 3).

Figura 3. Expresión oral por tipo de centro

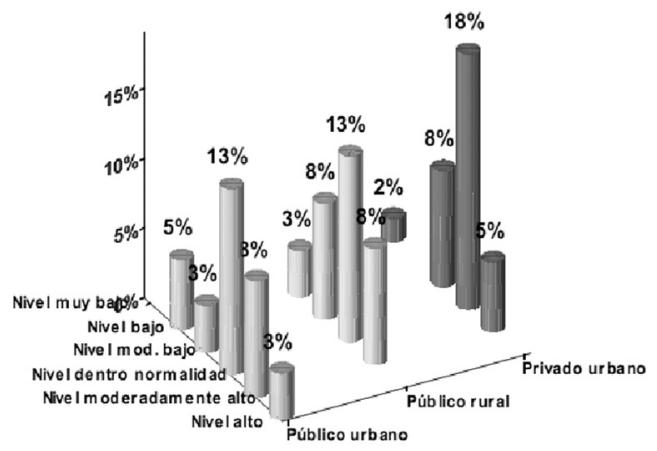


Sin embargo, entre los niveles superiores, la proporción de alumnos desciende hasta el 5\% para los centros privados urbanos, en el nivel moderadamente alto. Aquí, se invierte la tendencia anterior, y los públicos rurales y públicos urbanos se sitúan en el $8 \%$ del total de la muestra, por encima de los privados. Son muy pocos los casos recogidos en el nivel alto (3\%), y se encuentran dentro del grupo de los centros públicos urbanos.

Agrupando los resultados obtenidos en los niveles menos destacados (nivel muy bajo + nivel bajo + nivel moderadamente bajo), los centros públicos rurales (II\%) y los privados urbanos (I0\%) concentran unos porcentajes muy parecidos. Mientras tanto, en los públicos urbanos son menores los casos aparecidos, con un $8 \%$.

En conclusión, atendiendo solo a los mejores resultados (nivel dentro de la normalidad + nivel moderadamente alto + nivel alto), aunque los resultados de los públicos urbanos se encuentren en un $24 \%$, por encima de los privados urbanos (23\%) y de los públicos rurales (21\%), las diferencias son mínimas. Esto permite concluir sobre la semejanza en la competencia de expresión oral en los tres tipos de centros.

\section{Discusión y conclusiones}

Los resultados de este estudio permiten concluir que la expresión oral del alumnado se encuentra, principalmente, dentro de un nivel medio entre los sujetos estudiados. Resulta preocupante que la cuarta parte del total son alumnos que presentan déficits. Se tiende a relacionar ese nivel y carencias con una expresión oral que no suele ser aplicada en las aulas con mayor frecuencia, ya que las exigencias curriculares, las cuales, ante la obligación de impartir otros contenidos (gramática, vocabulario, etc.), exigen dilatar en el tiempo cualquier actividad de carácter oral. Existen en las aulas, ocasionalmente, situaciones en las que se restringen las intervenciones orales, porque los alumnos presentan déficits de expresión oral, por timidez, prejuicios u otras limitaciones. Pero estas razones no pueden servir de escudo para su omisión, pues pueden convertirse en un círculo vicioso. Para aprender a correr, primero es necesario andar. Y si el alumno no se ejercita en una expresión oral elemental en Lengua, se le estará 
anulando, también, la posibilidad de perder el miedo a preguntar dudas, a exponer con soltura la lección, etc. Es decir, no interviene, porque presenta déficits, pero tampoco se ejercita en aquello que le puede corregir sus déficits.

Precisamente, se tiende a ver la expresión oral como una actividad que puede resultar incontrolada en el aula, una situación que se le escapa de las manos al profesor. Sería conveniente, pues, aprovechar en beneficio del educador esas ganas de expresarse del alumno para introducir en el área de Lengua espacios de comunicación, de diálogo. En cualquier caso, siempre integrados dentro de actividades en las que el alumno tenga constancia de que está realizando un aprendizaje, y aplicando unas normas de diálogo que deberán ser respetadas. Es importante que el alumno adquiera cierta soltura para expresarse en público y aprenda a intercambiar ideas, ya que va a pasar una parte de su vida en un contexto académico. Sin olvidar, igualmente, que la técnica oral le resultará imprescindible, también, para el contexto social.

Se ha pasado, en muy pocos años, de un ciudadano parlante, a la figura del observador, con el uso de las pantallas (Funes, 2005). Este cambio ha provocado una relación entre la comunicación humana, la educación y los medios de comunicación, donde estos últimos no eliminan la comunicación, sino que más bien la favorecen (Cubillo y Torres, 2013; Fontcuberta, 2000). Integrar los medios para favorecer actividades lingüísticas en el aula (Vázquez y Martín, 20I4; Campos, Mendizábal y Gómez, 2012; Lara, Zayas, Alonso y Larequi, 2009) es imprescindible; pero esta sobreabundancia de medios no debe hacernos descuidar la didáctica de la comunicación oral, en sus tradicionales aspectos de diálogo y conversación.

Por otra parte, los resultados de este estudio demuestran, por género, una clara diferencia a favor de las alumnas en la prueba oral. Aunque no alcanzan el nivel de significatividad pretendido en este trabajo, se encuentran muy próximas a él. Estos resultados favorables a las alumnas, dentro del área de Lengua, se muestran en consonancia con aquellos otros que resultan en la mayoría de los estudios realizados, ya sean nacionales (Ministerio de Educación, 2014; 2010a; 2010b) como internacionales (Ministerio de Educación, 
20I3). Generalmente, en ellos se miden los aspectos que se refieren a la comprensión y expresión escrita, porque presentan menores dificultades de evaluación que la expresión oral. A pesar de tal hecho, algunos estudios propuestos para el nivel de Primaria (UNESCO, 2015; Ministerio de Educación, 2012) confirman, igualmente, un mejor rendimiento lingüístico de las alumnas en las pruebas realizadas. Incluso, en investigaciones en las que no se establecen diferencias significativas (Elices, Riveras, González y Crespo, 1990) se consigue apreciar, en lenguaje, una ligera tendencia favorable a las niñas. En este caso, puede considerarse relevante el dato, dado que el estudio se hizo para lo que hoy sería el primer curso de Primaria, donde ya, a tan temprana edad, se empezaría a notar la diferencia en la competencia lingüística.

Entre los motivos que pudieran explicar las diferencias de género en los resultados escolares del área de Lengua, la lectura puede hallarse entre las causas -o los efectos- de un mayor rendimiento de la competencia lingüística de las alumnas. Ellas se encuentran más interesadas en su práctica (Ministerio de Educación, 2015) $y$, consecuentemente, repercute en una mejor expresión oral, acompañada de una mayor riqueza léxica.

En cuanto al tipo de centro, no se aprecian diferencias en el rendimiento lingüístico entre alumnos de centros públicos y privados. Las diferencias resultantes no alcanzan distancias significativas. Estos resultados parecen refrendar los alcanzados por los centros escolares españoles en otros estudios (Ministerio de Educación, 2013). En todas las comunidades autónomas españolas los centros privados obtienen una puntuación superior a los centros públicos, pero esas diferencias son moderadas, especialmente, en Castilla y León.

Una posible razón que justifique la diferencia surgida entre las puntuaciones resultantes en dichos trabajos y las conseguidas en este, podría deberse a que aquéllos cuentan con un mayor número de sujetos investigados que esta; lo cual permitiría apreciar ese mínimo distanciamiento que en este no se observa.

Sin embargo, en España parece existir una cierta homogeneidad entre centros de titularidad pública y concertada. De modo que, si 
bien pueden producirse algunas diferencias de rendimiento entre ellos, estas pudieran atribuirse a los entornos sociales, económicos y culturales a los que tiene acceso el alumno y no tanto a una diferenciación de los currículos. Quizás, esto último se pueda apreciar más en países como Estados Unidos o Reino Unido, donde se aplica un tipo de educación más elitista, dependiendo de las señas de identidad del centro.

Para concluir se debe señalar que las destrezas orales requieren un “entrenamiento”. También, las escritas. Expresión oral y expresión escrita no pueden entenderse como compartimentos estancos. Más bien son complementarias y necesarias entre sí. Sería importante una práctica continuada de la expresión oral, desde la infancia hasta la conclusión de los estudios, adaptada a la edad del alumno (Quiles, 2006; Palou y Bosch, 2005; López y Encabo, 200I; Cañas, 2000), porque este un día dejará de serlo para integrarse definitivamente a la vida social y laboral y en ella se verá obligado a negociar y persuadir, reclamar, defender proyectos o proponer. En todos ellos tendrá que hacer un uso consciente y efectivo de su competencia lingüística. La escuela debe desarrollar en el alumno esta capacidad, pues la sociedad actual demanda con mucha frecuencia interacciones que requieren la expresión en público. Aquel alumno que no lo consiga podría encontrarse en la situación descrita a continuación:

Desgraciadamente en España esto es algo que no se enseña ni en las mejores escuelas, orientadas siempre a la evaluación escrita. Luego, vienen las sorpresas: uno acaba con su flamante título de Medicina, Derecho o Económicas y se planta en el salvaje mundo laboral con sus matrículas de honor a cuestas. Lo primero que le piden es una entrevista personal, y es ahí, precisamente ahí, donde se pone de manifiesto que el joven licenciado -a pesar de su brillante expediente- no sabe estar a la altura de las circunstancias (Merayo, 2000, p. 39).

La escuela propiciará diferentes situaciones en las que se desarrolle la necesidad de utilizar el lenguaje oral, la lectura y la escritura a través de diversos materiales, fuentes y recursos (Mata, Núñez y Rienda, 2015; Vilá y Castellá, 2014; Gómez, 2013; Badia y Vilá, 2009; Galera, 2009). Los fines han de ser variados, así como los interlocutores. 
Es así por lo que el Real Decreto I5I3/2006 determina que el desarrollo de la competencia lingüística, cuando el alumno haya finalizado la educación obligatoria, le llevará al dominio de la lengua oral y escrita en contextos múltiples y al uso funcional de, al menos, una lengua extranjera (Ministerio de Educación, 2006).

\section{Referencias bibliográficas}

Alférez, A. (2015). La comunicación eficaz del profesor: Una responsabilidad social. Opción, 3I (2), 34-49.

Aymerich, C. \& Aymerich, M. (197I). Expresión y arte en la escuela. I: La expresión en la escuela primaria. La expresión oral. Barcelona: Teide.

Badia, D. \& Vilá, M. (2009). Juegos de expresión oral y escrita. Barcelona: Graó.

Campos, A. F., Mendizábal, I. G. \& Gómez, M. D. M. P. (2012). Enseñanza de lenguas y TIC. Textos de Didáctica de la Lengua y la Literatura, 59, 63-72.

Cañas, J. (2000). Hablemos juntos. Barcelona: Octaedro.

Cruzata-Guzmán, R. \& Salazar-Salazar, M. (2012). La competencia comunicativa del maestro de la Educación Primaria. Ciencias Holguín, XVIII, I-I2.

Cubillo, M. D. \& Torres, J. J. (2013). ¿Mejoran las TIC los resultados académicos de los estudiantes españoles?, Extoikos, 9, 5I-58.

Doncel, J. \& Leena, M. (20I I). Las competencias básicas en la enseñanza. Fundamentación, enseñanza y evaluación. Sevilla: MAD.

Elices, J. A., Riveras, F., González, C. \& Crespo, M. A. (1990). El rendimiento escolar en función del sexo al inicio de la E.G.B. Revista Interuniversitaria de Formación del Profesorado, 8, I23-132.

Fontcuberta (de), M. (2000). Medios, comunicación humana y sociedad del conocimiento. Comunicar, I4, 25-34.

Funes, V. (2005). Espectadores, los alumnos del siglo XXI. Comunicar, 24, I05-II2.

Galera, F. (2009). La enseñanza de la lectura y la escritura: Teoría y práctica. Almería: Grupo Editorial Universitario.

Gimeno, J., Pérez, A. I.; Martínez, J. B.; Torres, J.; Angulo, F. \& Álvarez, J. M. (2008). Educar por competencias, ¿qué bay de nuevo? Madrid: Morata.

Gómez, I. (2013). La estimulación del lenguaje oral en Educación Primaria. Publicaciones Didácticas, 4I, 38-46. 
Gutiérrez, Y. \& Rosas, A. I. (2008). El lugar de la oralidad en la escuela: Exploraciones iniciales sobre las concepciones de los docentes. Infancias Imágenes, 7(I), 24-29.

Lara, T., Zayas, F., Alonso, N. \& Larequi, E. (2009). La competencia digital en el área de lengua. Barcelona: Octaedro.

Lomas, C. (Coord.) (2015). Fundamentos para una enseñanza comunicativa del lenguaje. Barcelona: Graó.

López, A. \& Encabo, E. (200I). Mejorar la comunicación en niños y adolescentes. Madrid: Pirámide.

Marco, B. (2008). Competencias básicas. Hacia un nuevo paradigma educativo. Madrid: Editorial Narcea.

Mata, J., Núñez, M. P. \& Rienda, J. (Coords. y Eds.) (2015). Didáctica de la lengua y la literatura. Madrid: Pirámide.

Merayo, A. (2000). Cómo aprender en el siglo de la información: Claves para una enseñanza más comunicativa. Comunicar, I4, 35-42.

Ministerio de Educación (2006). Real Decreto 1513/2006, de 7 de diciembre, por el que se establecen las enseñanzas mínimas de la Educación primaria. Recuperado de https://www.boe.es/boe/dias/2006/12/08/pdfs/A43053-43102. pdf

(2010a). Evaluación General de Diagnóstico. Educación Primaria. Cuarto curso. Informe de resultados. Madrid: Autor.

(2010b). Educación Primaria 2007. Evaluación general del sistema educativo. Madrid: Secretaría General Técnica.

(2012). PIRLS - TIMMS 2011. Estudio Internacional de progreso en comprensión lectora, matemáticas y ciencias. Informe español. Vols. I-II. IEA. Madrid: Instituto Nacional de Evaluación Educativa.

(2013). PISA 2012. Programa para la Evaluación Internacional de los Alumnos. Informe español. Madrid: Autor.

(2014). Sistema estatal de indicadores de la educación. Edición 2014. Madrid: Secretaría General Técnica.

(2015). Encuesta de bábitos y prácticas culturales 2014-2015. Madrid: Secretaría General Técnica.

Moya, J. \& Luengo, F. (Coords.). Asociación Proyecto Atlántida (20I I). Teoría y práctica de las competencias básicas. Barcelona: Graó.

Palou, J. \& Bosch, C. (Coord.) (2005). La lengua oral en la escuela: 10 experiencias didácticas. Barcelona: Graó. 
Pinker, S. (2003). El instinto del lenguaje. Madrid: Alianza Editorial.

Quiles, M. C. (2006). La comunicación oral. Propuestas didácticas para la Educación Primaria. Barcelona: Octaedro.

Segovia, J., Gallego, J. L. \& Rodríguez, A. (2013). Percepción del profesorado sobre la competencia comunicativa en estudiantes de Magisterio. Perfiles Educativos, 35(142), 54-74.

Serra, M., Serrat, E., Solé, R., Bel, A. \& Aparici, M. (2013). La adquisición del lenguaje. Barcelona: Ariel.

UNESCO (2015). Informe de resultados TERCE (Tercer Estudio Regional Comparativo y Explicativo). Recuperado de http://www.unesco.org/new/es/santiago/ terce/

Vázquez, E. \& Martín E. (20I4). Nuevas tendencias en la elaboración y utilización de materiales digitales para la enseñanza de lenguas. Madrid: McGraw-Hill.

Vilá, M. \& Castellá, J. M. (2014). 10 Ideas Clave. Enseñar la competencia oral en clase. Aprender a bablar en público. Barcelona: Graó.

Vila, M. \& Vila, I. (I994). Acerca de la enseñanza de la lengua oral. Comunicación, Lenguaje y Educación, 23, 45-54.

Vygotsky, L. (2012). Pensamiento y lenguaje. Barcelona: Paidós.

Zuccherini, R. (1992). Cómo educar la comunicación oral. Barcelona: Ceac. 